\title{
Spaghetti Meat Abnormality in Broilers: Current Understanding and Future Research Directions
}

\section{Giulia Baldi, Francesca Soglia and Massimiliano Petracci* \\ Department of Agricultural and Food Sciences, University of Bologna, Cesena, Italy}

Spaghetti meat (SM) is a recent muscular abnormality that affects the Pectoralis major muscle of fast-growing broilers. As the appellative suggests, this condition phenotypically manifests as a loss of integrity of the breast muscle, which appears soft, mushy, and sparsely tight, resembling spaghetti pasta. The incidence of SM can reach up to $20 \%$ and its occurrence exerts detrimental effects on meat composition, nutritional value, and technological properties, accounting for an overall decreased meat value and important economic losses related to the necessity to downgrade affected meats. However, due to its recentness, the causative mechanisms are still partially unknown and less investigated compared to other muscular abnormalities (i.e., White Striping and Wooden Breast), for which cellular stress and hypoxia caused by muscle hypertrophy are believed to be the main triggering factors. Within this scenario, the present review aims at providing a clear and concise summary of the available knowledge concerning SM abnormality and concurrently presenting the existing research gaps, as well as the potential future developments in the field.

Keywords: broilers, spaghetti meat, spaghetti breast, growth-related abnormalities, histology, meat quality, causative mechanisms

\section{INTRODUCTION}

About 10 years ago, the appearance of a novel cluster of muscular abnormalities affecting the Pectoralis major muscle of fast-growing broilers has alarmed the poultry industry. From there on, a growing body of literature has been focused on the etiology behind their appearance, implications on meat quality, as well as the attempts to mitigate their occurrence and narrow down the negative perception related to animal welfare (Griffin et al., 2018; Petracci et al., 2019; Baldi et al., 2020a). Within the industry vernacular, these myopathies are commonly called White Striping (WS), Wooden Breast (WB), and Spaghetti Meat (SM), each of which owning peculiar and distinctive traits from which their names originated. The first is indeed characterized by the occurrence of white lines running parallel to the muscle fibers on the surface of $P$. major muscle (Kuttappan et al., 2013b); the second manifests as a severe hardening of the pectoral muscle (Sihvo et al., 2014), while the third displays as a loss of integrity of the muscle fiber bundles composing the breast muscle itself, which appears mushy and sparsely tight (Baldi et al., 2018). Due to their alarming incidence rates and outstanding impact on meat quality, a large number of studies has been published on WS and WB abnormalities over the years, focusing on the understanding of their etiology, the molecular pathways potentially responsible for their occurrence as well as their implications on eating, nutritional, and technological properties of meat (Petracci et al., 2019). On the contrary, the information regarding SM is today still limited and only few studies have been published since 
its appearance (Table 1). Thus, the present review aims at providing a clear and succinct summary of the available knowledge concerning SM condition and presenting the current research challenges as well as the potential future developments in the field.

\section{UP-TO-DATE KNOWLEDGE}

\section{Morphological Characteristics and Incidence Levels}

Spaghetti Meat defect was first recounted in 2015 with the name of "Mushy Breast" and described as a myopathy causing the loss of muscle integrity of the $P$. major muscle of fastgrowing chickens (Bilgili, 2015). Later on, this condition has been commonly recognized with the name of "Spaghetti Meat" or "Spaghetti Breast" since, as the appellative suggests, it phenotypically manifests with the detachment of the fiber bundles composing the pectoral muscle, which appears soft, mushy, and sparsely thigh, resembling spaghetti pasta. Since the severity of SM defect might be variable, Sirri et al. (2016) proposed a classification criterion based on a three-score scale. Indeed, depending on the severity score, the occurrence of SM can be detected either palpably, due to the soft and stringy structure perceived by pinching the muscle on its surface, or visually, due to the expanded superficial lacerations (Figure 1). As will be further explained (see section "Implications on meat quality and practical solutions"), meats severely affected by SM are usually downgraded and incorporated into the formulation of further processed products, while moderate cases can be marketed for fresh retailing. Generally, SM condition mostly manifests in the ventro-cranial portion of the fillet, but there is growing evidence that also the caudal section and, occasionally, leg muscles might be affected as well. Recently, a sporadic occurrence of SM defect has been also signaled on the pectoral muscles of commercial turkeys, even though no information is yet available concerning possible similarities with the same condition reported for broilers (Zampiga et al., 2020).

Data concerning the incidence rates of SM condition are limited and sometimes contradictory, likely due to both the variation of classification criteria among the abattoirs and the complication related to the concurrent presence of other myopathies within the same muscle, since SM can be comorbid with WB and, more likely, with WS (Baldi et al., 2018; Bailey et al., 2020). However, an Italian survey carried out on 16,000 breasts reported that around $21 \%$ of samples were affected by SM (Baldi et al., 2020a), while a Brazilian study evaluated about 10\% of SM fillets on a total of 5,580 breasts (Montagna et al., 2019). While it has been widely recognized that the incidence rates of WS and WB significantly upsurge with higher growth rate, breast yield as well as body weight and age at slaughter of birds (Kuttappan et al., 2013a, 2016; Papah et al., 2018), this trend has not been fully confirmed for SM. However, Pascual et al. (2020) intriguingly found significantly higher rates of SM in females rather than males (25.0\% vs. $3.1 \%$, respectively), contrarily to what observed for WS and WB, whose incidence levels were found to be greater in males regardless the slaughter weight (Lorenzi et al., 2014; Trocino et al., 2015). To the best of our knowledge, the reasons for the higher incidence of SM condition in female individuals are still unknown, albeit a recent study highlighted an increased expression of genes related to connective tissue proliferation in male broilers, making them more prone than females to develop WB condition (Brothers et al., 2019). However, a possible role of a different hormonal response (e.g., insulin growth factor, somatotropic hormone, myostatin, etc.) regulating metabolism, protein synthesis, patterns of intramuscular fat deposition, and intracellular signaling during the development of myopathic conditions might be also speculated.

\section{Histological Traits}

Microscopic investigations performed on SM muscles highlighted several histologic features commonly reported also for WS and WB, such as nuclei internalization, inflammatory cells infiltration, necrosis, fiber lysis, and concurrent presence of small regenerative fibers combined with abnormal ones showing larger diameter (Baldi et al., 2018). On the other hand, the distinctive microscopic feature of SM condition is the progressive rarefaction of the endomysial and perimysial connective tissue, that likely leads to the detachment of the muscle fibers from each others (Baldi et al., 2018). Moreover, histological observations highlighted the presence of small and thin fibers, interspersed by immature/newly deposited connective tissue (Baldi et al., 2018; Sanden et al., 2021). The presence of these fibers, distinguished by a remarkably reduced cross-sectional area, suggests the ongoing of regenerative processes taking place in the muscle, as a natural response mechanism to cell necrosis (Mazzoni et al., 2020). However, a different phase of cell regeneration can be hypothesized when comparing SM and WS samples' immunoreactivity to vimentin, suggesting a different progression of the regenerative processes (Soglia et al., 2020). A recent study also investigated the distribution of collagen type III and its precursor (i.e., procollagen type III) in SM samples since, being involved in fibrillogenesis, their possible altered deposition might have a key role in the occurrence of muscular abnormalities. In this regard, the findings highlighted an altered immunoreactivity to procollagen type III in SM samples, suggesting a compromised collagen turnover and synthesis (Mazzoni et al., 2020).

\section{Implications on Meat Composition}

The degenerative processes and repair mechanisms taking place in the muscles affected by growth-related abnormalities (e.g., inflammatory processes, necrosis, fibrosis, lipidosis, etc.) inevitably alter the proximate composition of meat and, as a direct consequence, negatively affect its nutritional value (Petracci et al., 2019). In detail, as reported by Baldi et al. $(2018,2019)$, the occurrence of SM is associated with a remarkable relatively reduction of protein content ($10 \%$, when compared to unaffected muscles) coupled with a concurrent increase in fat and moisture levels $(+21.8$ and + $3.0 \%$, respectively). The same authors reported a lower content of EPA and DHA in meat affected by SM, likely accountable for a different expression of the genes encoding for $\Delta 5$ and $\Delta 6$ desaturases, as previously hypothesized by Soglia et al. (2016a). As concern mineral profile, a study carried out by Tasoniero et al. (2020) reported greater calcium and sodium levels in SM samples if compared to unaffected ones, speculating a possible connection 
TABLE 1 | Chronological sequence of available published studies concerning spaghetti meat condition and relative addressed topics.

\begin{tabular}{|c|c|c|c|c|c|c|c|c|}
\hline \multirow[b]{2}{*}{ References } & \multirow[b]{2}{*}{$\begin{array}{l}\text { Implications of } \\
\text { genetic and } \\
\text { environmental } \\
\text { factors on } \\
\text { incidence levels }\end{array}$} & \multirow[b]{2}{*}{$\begin{array}{c}\text { Detection } \\
\text { tools }\end{array}$} & \multirow[b]{2}{*}{$\begin{array}{c}\text { Meat } \\
\text { processing } \\
\text { solutions }\end{array}$} & \multicolumn{4}{|c|}{ Examined traits } & \multirow[b]{2}{*}{$\begin{array}{c}\text { Technological } \\
\text { quality }\end{array}$} \\
\hline & & & & Histology & $\begin{array}{l}\text { Proximate } \\
\text { composition }\end{array}$ & $\begin{array}{c}\text { Protein } \\
\text { and/or amino } \\
\text { acid profile }\end{array}$ & Collagen & \\
\hline Sirri et al., 2016 & $\bullet$ & & & & & & & \\
\hline Baldi et al., 2018 & & & $\bullet$ & $\bullet$ & $\bullet$ & $\bullet$ & & $\bullet$ \\
\hline Córdova-Noboa et al., 2018 & $\bullet$ & & & & & & & \\
\hline Zampiga et al., 2018 & $\bullet$ & & & & & & & \\
\hline Baldi et al., 2019 & & & - & & $\bullet$ & 0 & - & - \\
\hline Montagna et al., 2019 & $\bullet$ & & & & & & & \\
\hline Soglia et al., 2019a & & & - & & & - & & - \\
\hline Mazzoni et al., 2020 & & & & ○ & & & ○ & \\
\hline Bailey et al., 2020 & $\bullet$ & & & & & & & \\
\hline Campo et al., 2020 & & $\bullet$ & & & & & & $\bullet$ \\
\hline Morey et al., 2020 & & - & & & & & & \\
\hline Pascual et al., 2020 & $\bullet$ & & & & & & & \\
\hline Soglia et al., 2020 & & & & & & 0 & 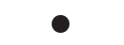 & \\
\hline Tasoniero et al., 2020 & & & & & $\bullet$ & 0 & 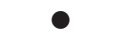 & $\bullet$ \\
\hline Yu et al., 2020 & & & & & & & & ○ \\
\hline Pascual et al., 2021 & & & & & $\bullet$ & & & $\bullet$ \\
\hline Sanden et al., 2021 & & & & 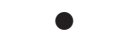 & & & $\bullet$ & \\
\hline
\end{tabular}

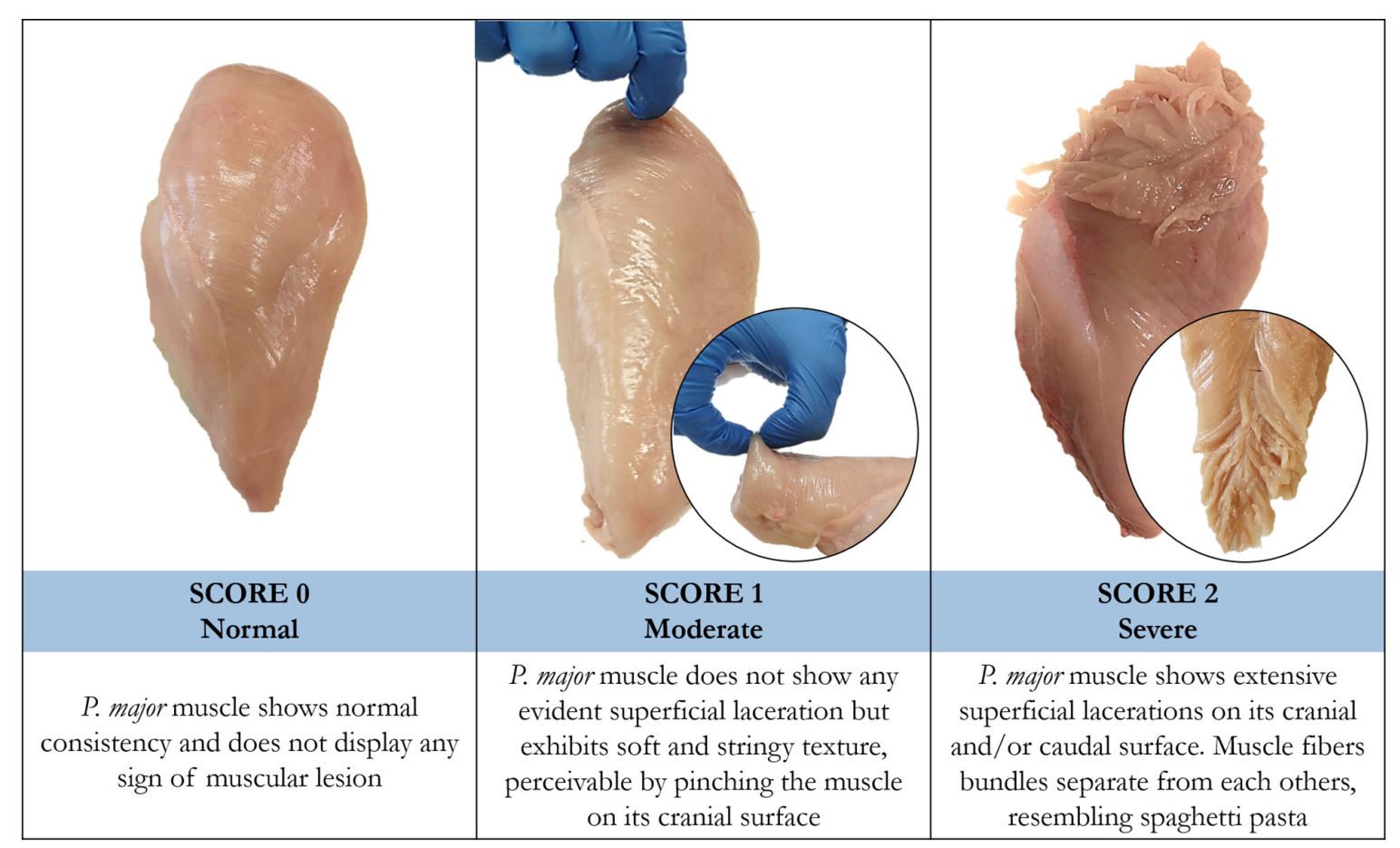

FIGURE 1 | Representative images of chicken breast fillets with normal (score 0), moderate (score 1) and severe (score 2) degrees of Spaghetti Meat.

between cation homeostasis disturbances and the appearance of pathological mechanisms leading to cell injury development. Intriguingly, the analysis of the available literature highlighted that SM samples possess analogous amounts of total and soluble collagen of unaffected breasts (Baldi et al., 2018; Campo et al., 2020; Tasoniero et al., 2020). On the other hand, Baldi et al. (2019) found lower hydroxylysylpyridinoline contents in muscles affected by SM, suggesting a feeble collagen cross-linking (i.e., 
presence of immature collagen) and corroborating what found from previous histological observations (see section "Histological traits"). Accordingly, following investigations through polarized FTIR-spectroscopy, Sanden et al. (2021) reported that SM muscles possess thin, loose and immature collagen fiber bundles, which are also poorly packed. The same authors also mentioned that SM shows a higher amount of glycosaminoglycans in perimysial connective tissue, corroborating the hypothesis put forward by Papah et al. (2018) of a shift in glucose flux toward the synthesis of molecules composing the extracellular matrix in myopathic muscles.

\section{Implications on Meat Quality and Practical Solutions}

Albeit only few studies have been published concerning the implications of SM condition on meat quality (see Table 1), its occurrence seems to affect meat technological properties and functionality to a lesser extent if compared to WS and WB (Baldi et al., 2019; Pascual et al., 2021). Overall, the occurrence of $\mathrm{SM}$ is associated with higher meat yellowness and ultimate $\mathrm{pH}$ values (Baldi et al., 2018; Soglia et al., 2019a; Tasoniero et al., 2020). While $\mathrm{Yu}$ et al. (2020) did not find any difference in water holding capacity assessed on fresh, frozen, and cooked SM samples, Pascual et al. (2021) reported greater drip and cooking losses in SM samples compared to normal ones. The latter result corroborates the outcomes obtained through time-domain nuclear magnetic resonance analysis, which evidenced increased amounts of bound and extra-myofibrillar water fractions in SM fillets (Soglia et al., 2019a). As suggested by the longer relaxation times, the abovementioned water fractions are concurrently less tightly bound to the muscle tissue, highlighting an altered water distribution and mobility within SM muscles and, thus, an impaired ability to hold constitutional water (Baldi et al., 2019). This hypothesis is further supported by the results obtained by Tasoniero et al. (2020), who found a remarkable reduction of myofibrillar protein solubility, salted-induced water uptake, final yield as well as emulsion stability in SM fillets if compared to unaffected ones. Intriguingly, SM breasts did not display any upsurge in protein carbonylation levels (Baldi et al., 2018; Soglia et al., 2019a), suggesting that the reduced water holding capacity is not imputable to the ongoing of oxidative processes occurring at the expenses of muscular proteins. Thus, overall, this phenomenon can be explained by further mechanisms which essentially share the same underpinning factor. Precisely, the complete re-organization of muscle architecture following myodegeneration and necrosis leads to a reduction in the number of functional myofibrils and, therefore, to a lower potential of the muscle to bind water (Soglia et al., 2016a; Tasoniero et al., 2017). Indeed, the impaired water holding capacity of SM samples might be the direct consequence of their reduced protein solubility, probably due to the ongoing of protein degradation processes as evidenced by both the higher myofibrillar fragmentation index of SM muscles if compared to unaffected ones (Baldi et al., 2018; Tasoniero et al., 2020) and the greater concentration of free amino acids deriving from the breakdown of muscular proteins (Soglia et al., 2019a). In support of this, electrophoretic analysis evidenced an increased number of high molecularweight bands in SM samples, presumably originating from the degenerative processes associated with the occurrence of muscular abnormalities (Baldi et al., 2018).

As concern texture, compression forces obtained for raw meat did not evidence any difference between SM samples and their unaffected counterparts (Baldi et al., 2019). However, after the cooking process, SM fillets showed a softer texture following compression, Meullenet-Owens Razor Shear as well as AlloKramer tests (Baldi et al., 2019; Pascual et al., 2021), probably as a result of their reduced collagen cross-linking degree. In addition, a number of studies have been carried out to investigate whether SM lesions display intra-fillet variations, revealing that the occurrence of this condition mainly alters meat composition and quality traits of the superficial layer of the muscle, while the deep one is almost unaffected (Baldi et al., 2019; Tasoniero et al., 2020; Yu et al., 2020). However, to the best of our knowledge, any published study specifically speculates about those divergences. Nevertheless, intensive selection practices carried out over years permitted to accomplish a remarkable development of the breast muscle, so much so that the impressive thickness of its cranial section might compress the pectoral artery, reducing muscular oxygenation (Soglia et al., 2021). Thus, a feasible hypothesis for the abovementioned intra-fillet variations may rely on a different blood flow between the surface of the muscle, phenotypically exhibiting the defect, and its deep counterpart. The first, being thicker and likely less oxygenated compared to the latter, might be more prone to develop inflammatory processes due to the weakened blood supply and impaired displacement of metabolic end-products, thus resulting in the advancing of hypoxic conditions which are believed to be the triggering factor for the manifestation of growth-related abnormalities (Abasht et al., 2016; Malila et al., 2019; Greene et al., 2020). Indeed, as observed at least for WS and WB, the severity of the histological lesions gradually weakens when moving from the antero-ventral to the antero-dorsal section of $P$. major muscle (Soglia et al., 2016b; Clark and Velleman, 2017; Baldi et al., 2020b).

The abovementioned detrimental effects of SM condition on meat quality may account for economic losses related to decreased yields during processing. Generally speaking, meats severely affected by SM are usually downgraded and incorporated into the formulation of further processed products, while moderate cases can be marketed for fresh retailing (Petracci et al., 2019). However, the growing consumers' demand for thin-sliced chicken breast meat has made the occurrence of SM particularly challenging for the processing industry since, being SM often detectable only after the slicing process, the amount of downgraded/discarded meat could be even exacerbated. Thus, being the in-line detection of abnormal meat a relevant matter especially for the meat packing plants, Morey et al. (2020) recently proposed the feasibility of bioelectrical impedance analysis to identify SM breasts, while Campo et al. (2020) suggested the analysis of color reflectance as a possible tool to detect those breasts affected by any degree of WS and SM in the processing line. Aside from this, it is noteworthy to mention that SM myopathy cannot be noticed in the living animal, contrarily to WB condition whose presence can be detected by palpating the 
breast of the live bird and lifting up its wings to assess the ability to achieve back-to-back wing contact (Hosotani et al., 2020). This might represent a useful tool for breeder companies to exclude birds showing WB defect from the pedigree lines, even though this would entail the possible risk to indirectly select individuals showing SM condition. Indeed, evidence suggests that a reduction of $\mathrm{WB}$ occurrence in some flocks is sometimes associated with a concurrent increase in SM condition levels.

Thus, given the detrimental impact of SM condition and, more generally, of growth-related abnormalities on chicken meat quality and the economy of poultry meat producers, the pursuit for potential solutions to mitigate these undesirable implications is calling the attention of the scientific community and have been recently summarized in the reviews written by Barbut (2019), Petracci et al. (2019), and Baldi et al. (2020a). Considering the current lack of efficient animals' nutrition and management strategies to reduce SM defect without affecting slaughter performances (Sirri et al., 2016; Córdova-Noboa et al., 2018; Zampiga et al., 2018), to date, the most promising approach seems to develop processing solutions aimed at reducing the implications on the final quality of meat. Within this context, the most practical solution so far is represented by the separation of the superficial and deep sections of the fillets, addressing the first for the manufacture of processed products, while the latter for fresh retailing. Indeed, as reported in the previous paragraph, the occurrence of SM negatively impacts only the surface of the muscle, thus allowing to downgrade a reduced portion of the fillet. As previously suggested for WB (Brambila et al., 2017; Xing et al., 2017; Chen et al., 2018), downgraded meat could be then included in the formulation of ground or finely comminuted meat products, where the addition of functional ingredients (i.e., starches, phosphates, hydrocolloids, etc.) could mask its impaired technological properties (Petracci et al., 2019). Moreover, since it has been reported that freezing and subsequent thawing - do not result in any further worsening of SM meat quality traits (Soglia et al., 2019a), downgraded fillets can be frozen and afterward included in the formulation of processed products, guaranteeing a greater flexibility for the poultry processing industry.

\section{CURRENT CHALLENGES AND POTENTIAL FUTURE DEVELOPMENTS}

Considering the recentness of SM condition, the mechanisms underpinning its appearance are still partially unknown and less investigated compared to WB and WS. However, the same histological alterations shared among WB, WS, and SM fillets suggest a common network of causative mechanisms responsible for their occurrence, in which cellular stress and hypoxia caused by muscle hypertrophy induced by selection - play a key role (Sihvo et al., 2018; Young and Rasmussen, 2020; Soglia et al., 2021). Considering their common histological traits, one of the most compelling challenges for the scientific community is to determine the reason why growth-related myopathies phenotypically manifest differently. Within this context, Soglia et al. (2020) recently speculated that the distinctive phenotype of WB, WS, and SM meat could be associated, at least partially, to the synthesis of vimentin, a protein considered a marker of muscle fiber regeneration and directly involved in the coordination of fibroblast proliferation (Cheng et al., 2016). In more detail, it has been suggested that the lack of correspondence between the up-regulated gene encoding for vimentin and its encoded protein found in SM muscles might be responsible for an altered distribution of fibroblasts in the perimysial compartment, which would ultimately result in a progressive rarefaction of the connective tissue, a typical trait of SM myopathy (Soglia et al., 2020).

Otherwise, the distinctive phenotype of SM has been also thought to be associated with an excessive build-up of lactic acid in the muscle, leading to the inhibition of protein synthesis regulating collagen development (Anton et al., 2019). On the other hand, contrarily to what previously observed for WS and WB (Radaelli et al., 2017), it must be pointed out that the low heritability levels of SM may indicate the impact of non-genetic factors on the variance of the myopathy traits (Bailey et al., 2020). Indeed, the weak correlation between the incidence of SM condition and animals' growth rate, age, and weight at slaughter might hint at other "contributing factors" related to slaughtering operations (e.g., scalding, de-feathering, chilling, post-mortem deboning time and technology, etc.) which can exploit a role in the manifestation of SM condition or, at least, in the worsening of its severity level. Indeed, a recent technical report highlighted that the incidence of SM was found to be up to $50 \%$ higher in carcasses subjected to slow carcass chilling compared to their fast-cooled counterparts (Anton et al., 2019). The authors justified this phenomenon with an excessive accumulation of lactic acid in the muscle when the carcass is still warm. This would stimulate the activity of proteases responsible for the degradation of connective tissue and subsequent excessive softening of the meat (Anton et al., 2019). However, from the analysis of the available literature, no research aimed at establishing the effect of chilling conditions on the occurrence of SM is available yet, thus being a stimulating starting point for future investigations. Another appealing challenge for the poultry processors could be the understanding of the possible role of scalding and plucking procedures in a further worsening of the consistency of those fillets exhibiting a mild level of muscle destructuration. Indeed, given the presence of weakened perimysial connective tissue in SM samples (Baldi et al., 2018), it could be hypothesized that scalding temperatures combined with an aggressive de-feathering could likely worsen an already compromised muscular structure, making the muscle fibers more prone to be torn apart during handling and fileting. In addition, a new emerging quality issue phenotypically resembling SM condition was recently found to affect the $P$. minor muscles of broiler chickens. This defect is named "gaping" and has been examined for the first time in the study of Soglia et al. (2019b), who suggested that its occurrence is especially related to peri-mortem factors as well as slaughtering procedures. Notwithstanding, further studies should be performed to establish whether a relationship exists between the occurrence of gaping defect in the $P$. minor muscles and SM in their corresponding major counterparts.

In conclusion, considering the available knowledge and the existing research gaps about SM myopathy, the focus of future investigations might be directed into the unraveling of the role 
of peri-mortem procedures in the development of this condition, as well as the underpinning factors that make the incidence of SM higher in female individuals and inversely correlated to the manifestation of WB condition.

\section{AUTHOR CONTRIBUTIONS}

GB was the primary writer and performed most of the literature search. FS and MP supported the redaction of all sections. All

\section{REFERENCES}

Abasht, B., Mutryn, M. F., Michalek, R. D., and Lee, W. R. (2016). Oxidative stress and metabolic perturbations in wooden breast disorder in chickens. PLoS One 11:e0153750. doi: 10.1371/journal.pone.0153750

Anton, P., Avendanõ, S., Biley, R., Bilgili, S., Canela, L., and Corzo, A. (2019). Breast muscle myopathies Aviagen. Available online at: http://en.aviagen.com/assets/Tech_Center/Broiler_Breeder_Tech_Articles/ English/Breast-Muscle-Myopathies-2019-EN.pdf\%0A (accessed April 20, 2021).

Bailey, R. A., Souza, E., and Avendano, S. (2020). Characterising the influence of genetics on breast muscle myopathies in broiler chickens. Front. Physiol. 11:1041. doi: 10.3389/fphys.2020.01041

Baldi, G., Soglia, F., Laghi, L., Tappi, S., Rocculi, P., Tavaniello, S., et al. (2019). Comparison of quality traits among breast meat affected by current muscle abnormalities. Food Res. Int. 115, 369-376. doi: 10.1016/j.foodres.2018.11.020

Baldi, G., Soglia, F., Mazzoni, M., Sirri, F., Canonico, L., Babini, E., et al. (2018). Implications of white striping and spaghetti meat abnormalities on meat quality and histological features in broilers. Animal 12, 164-173. doi: 10.1017/ S1751731117001069

Baldi, G., Soglia, F., and Petracci, M. (2020a). Current status of poultry meat abnormalities. Meat Muscle Biol. 4, 1-7. doi: 10.22175/mmb.9503

Baldi, G., Yen, C.-N., Daughtry, M. R., Bodmer, J., Bowker, B., Zhuang, H., et al. (2020b). Exploring the factors contributing to the high ultimate ph of broiler pectoralis major muscles affected by wooden breast condition. Front. Physiol 11:343. doi: 10.3389/fphys.2020.00343

Barbut, S. (2019). Recent myopathies in broiler's breast meat fillets. Worlds. Poult. Sci. J. 75, 559-582. doi: 10.1017/S0043933919000436

Bilgili, S. (2015). Broiler chicken myopathies IV. Stringy/mushy breast. in Worthwhile Operational Guidelines and Suggestion. Available online at: http: //poul.auburn.edu/wp-content/uploads/sites/13/2015/11/WOGS-FEB15.pdf (accessed April 20, 2021).

Brambila, G. S., Chatterjee, D., Bowker, B., and Zhuang, H. (2017). Descriptive texture analyses of cooked patties made of chicken breast with the woody breast condition. Poult. Sci. 96, 3489-3494. doi: 10.3382/ps/pex118

Brothers, B., Zhuo, Z., Papah, M. B., and Abasht, B. (2019). RNA-seq analysis reveals spatial and sex differences in pectoralis major muscle of broiler chickens contributing to difference in susceptibility to wooden breast disease. Front. Physiol. 10:764. doi: 10.3389/fphys.2019.00764

Campo, M. D. M., Mur, L., Guerrero, A., Barahona, M., Resconi, V. C., Magalhaes, D. R., et al. (2020). Differentiating breast myopathies through color and texture analyses in broiler. Foods 9:824. doi: 10.3390/foods9060824

Chen, H., Wang, H. H., Qi, J., Wang, M., Xu, X., and Zhou, G. (2018). Chicken breast quality - normal, pale, soft and exudative (PSE) and woody - influences the functional properties of meat batters. Int. J. Food Sci. Technol. 53, 654-664. doi: $10.1111 /$ ijfs. 13640

Cheng, F., Shen, Y., Mohanasundaram, P., Lindström, M., Ivaska, J., Ny, T., et al. (2016). Vimentin coordinates fibroblast proliferation and keratinocyte differentiation in wound healing via tgf- $\beta$-slug signaling. Proc. Natl. Acad. Sci. U.S.A. 113, E4320-E4327. doi: 10.1073/pnas.1519197113

Clark, D. L., and Velleman, S. G. (2017). Spatial influence on breast muscle morphological structure, myofiber size, and gene expression associated with the wooden breast myopathy in broilers. Poult. Sci. 95, 2930-2945. doi: 10.3382/ps/ pew243 authors listed have made a substantial, direct and intellectual contribution to the work, and approved it for publication.

\section{FUNDING}

This work has been partially supported by the PRIN project "Use of local chicken breeds in alternative production chain: welfare, quality and sustainability" (Prot. 2017S229WC) funded by the Italian Ministery of Research (MIUR).

Córdova-Noboa, H. A., Oviedo-Rondón, E. O., Sarsour, A. H., Barnes, J., Ferzola, P., Rademacher-Heilshorn, M., et al. (2018). Performance, meat quality, and pectoral myopathies of broilers fed either corn or sorghum based diets supplemented with guanidinoacetic acid. Poult. Sci. 97, 2479-2493. doi: 10. 3382/ps/pey096

Greene, E., Cauble, R., Dhamad, A. E., Kidd, M. T., Kong, B., Howard, S. M., et al. (2020). Muscle metabolome profiles in woody breast-(un)affected broilers: effects of quantum blue phytase-enriched diet. Front. Vet. Sci. 7:458. doi: 10. 3389/fvets. 2020.00458

Griffin, J. R., Moraes, L., Wick, M., and Lilburn, M. S. (2018). Onset of white striping and progression into wooden breast as defined by myopathic changes underlying Pectoralis major growth. Estimation of growth parameters as predictors for stage of myopathy progression. Avian Pathol. 47, 2-13. doi: 10.1080/03079457.2017.1356908

Hosotani, M., Kawasaki, T., Hasegawa, Y., Wakasa, Y., Hoshino, M., Takahashi, N., et al. (2020). Physiological and pathological mitochondrial clearance is related to pectoralis major muscle pathogenesis in broilers with wooden breast syndrome. Front. Physiol. 11:579. doi: 10.3389/fphys.2020. 00579

Kuttappan, V. A., Brewer, V. B., Mauromoustakos, A., McKee, S. R., Emmert, J. L., Meullenet, J. F., et al. (2013a). Estimation of factors associated with the occurence of white striping in broilr breast fillets. Poult. Sci. 92, 811-819. doi: $10.3382 /$ ps.2012-02506

Kuttappan, V. A., Hargis, B. M., and Owens, C. M. (2016). White striping and woody breast myopathies in the modern poultry industry: a review. Poult. Sci. 95, 2724-2733. doi: 10.3382/ps/pew216

Kuttappan, V. A., Shivaprasad, H. 1, Shaw, D. P., Valentine, B. A., Hargis, B. M., Clark, F. D., et al. (2013b). Pathological changes associated with white striping in broiler breast muscles. Poult. Sci 92, 331-338. doi: 10.3382/ps.201202646

Lorenzi, M., Mudalal, S., Cavani, C., and Petracci, M. (2014). Incidence of white striping under commercial conditions in medium and heavy broiler chickens in Italy. J. Appl. Poult. Res. 23, 754-758. doi: 10.3382/japr.201400968

Malila, Y., Thanatsang, K., Arayamethakorn, S., Uengwetwanit, T., Srimarut, Y., Petracci, M., et al. (2019). Absolute expressions of hypoxia-inducible factor-1 alpha (HIF1A) transcript and the associated genes in chicken skeletal muscle with white striping and wooden breast myopathies. PLoS One 14:e220904. doi: 10.1371/journal.pone.0220904

Mazzoni, M., Soglia, F., Petracci, M., Sirri, F., Lattanzio, G., and Clavenzani, P. (2020). Fiber metabolism, procollagen and collagen type III immunoreactivity in broiler pectoralis major affected by muscle abnormalities. Animals 10:1081. doi: 10.3390/ani10061081

Montagna, F. S., Garcia, G., Nääs, I. A., Lima, N. D. S., and Caldara, F. R. (2019). Practical Assessment of spaghetti breast in diverse genetic strain broilers reared under different environments. Rev. Bras. Cienc. Avic 21:eRBCA-2019-0759. doi: 10.1590/1806-9061-2018-0759

Morey, A., Smith, A. E., Garner, L. J., and Cox, M. K. (2020). Application of bioelectrical impedance analysis to detect broiler breast filets affected with woody breast myopathy. Front. Physiol. 11:808. doi: 10.3389/fphys.2020.00808

Papah, M. B., Brannick, E. M., Schmidt, C. J., and Abasht, B. (2018). Gene expression profiling of the early pathogenesis of wooden breast disease in commercial broiler chickens using RNA-sequencing. PLoS One 13:e0207346. doi: 10.1371/journal.pone.0207346 
Pascual, A., Trocino, A., Birolo, M., Cardazzo, B., Bordignon, F., Ballarin, C., et al. (2020). Dietary supplementation with sodium butyrate: growth, gut response at different ages, and meat quality of female and male broiler chickens. Ital. J. Anim. Sci. 19, 1135-1146. doi: 10.1080/1828051X.2020.1824590

Pascual, A., Trocino, A., Susta, L., and Barbut, S. (2021). Comparing three textural measurements of chicken breast fillets affected by severe wooden breast and spaghetti meat. Ital. J. Anim. Sci. 20, 465-471. doi: 10.1080/1828051X.2021. 1893134

Petracci, M., Soglia, F., Madruga, M., Carvalho, L., Ida, E., and Estévez, M. (2019). Wooden-breast, white striping, and spaghetti meat: causes, consequences and consumer perception of emerging broiler meat abnormalities. Compr. Rev. Food Sci. Food Saf. 18, 565-583. doi: 10.1111/1541-4337.12431

Radaelli, G., Piccirillo, A., Birolo, M., Bertotto, D., Gratta, F., Ballarin, C., et al. (2017). Effect of age on the occurrence of muscle fiber degeneration associated with myopathies in broiler chickens submitted to feed restriction. Poult. Sci. 96, 309-319. doi: 10.3382/ps/pew270

Sanden, K. W., Bocker, U., Ofstad, R., Pedersen, M. E., Host, V., Afseth, N. K., et al. (2021). Characterization of collagen structure in normal, wooden breast and spaghetti meat chicken fillets by FTIR microspectroscopy and histology. Foods 10:548.

Sihvo, H. K., Airas, N., Lindén, J., and Puolanne, E. (2018). Pectoral vessel density and early ultrastructural changes in broiler chicken wooden breast myopathy. J. Comp. Pathol. 161, 1-10. doi: 10.1016/j.jcpa.2018.04.002

Sihvo, H. K., Immonen, K., and Puolanne, E. (2014). Myodegeneration with fibrosis and regeneration in the pectoralis major muscle of broilers. Vet. Pathol. 51, 619-623. doi: 10.1177/0300985813497488

Sirri, F., Maiorano, G., Tavaniello, S., Chen, J., Petracci, M., and Meluzzi, A. (2016). Effect of different levels of dietary zinc, manganese, and copper from organic or inorganic sources on performance, bacterial chondronecrosis, intramuscular collagen characteristics, and occurrence of meat quality defects of broiler chickens. Poult. Sci. 95, 1813-1824. doi: 10.3382/ps/pew064

Soglia, F., Laghi, L., Canonico, L., Cavani, C., and Petracci, M. (2016a). Functional property issues in broiler breast meat related to emerging muscle abnormalities. Food Res. Int. 89, 1071-1076. doi: 10.1016/j.foodres.2016. 04.042

Soglia, F., Mazzoni, M., Zappaterra, M., Di Nunzio, M., Babini, E., Bordini, M., et al. (2020). Distribution and expression of vimentin and desmin in broiler pectoralis major affected by the growth-related muscular abnormalities. Front. Physiol 10:1581. doi: 10.3389/fphys.2019.01581

Soglia, F., Mudalal, S., Babini, E., Di Nunzio, M., Mazzoni, M., Sirri, F., et al. (2016b). Histology, composition, and quality traits of chicken Pectoralis major muscle affected by wooden breast abnormality. Poult. Sci. 95, 651-659. doi: $10.3382 / \mathrm{ps} / \mathrm{pev} 353$

Soglia, F., Petracci, M., Davoli, R., and Zappaterra, M. (2021). A critical review of the mechanisms involved in the occurrence of growth-related abnormalities affecting broiler chickens breast muscle. Poultry Sci. 100:101180. doi: 10.1016/j. psj.2021.101180

Soglia, F., Silva, A. K., Lião, L. M., Laghi, L., and Petracci, M. (2019a). Effect of broiler breast abnormality and freezing on meat quality and metabolites assessed by 1 H-NMR spectroscopy. Poult. Sci. 98, 7139-7150. doi: 10.3382/ps/ pez514

Soglia, F., Silva, A. K., Tappi, S., Lião, L. M., Rocculi, P., Laghi, L., et al. (2019b). Gaping of pectoralis minor muscles: Magnitude and characterization of an emerging quality issue in broilers. Poult. Sci. 98, 6194-6204. doi: 10.3382/ps/ pez418

Tasoniero, G., Bertram, H. C., Young, J. F., Dalle Zotte, A., and Puolanne, E. (2017). Relationship between hardness and myowater properties in Wooden Breast affected chicken meat: a nuclear magnetic resonance study. LWT 86, 20-24. doi: 10.1016/J.LWT.2017.07.032

Tasoniero, G., Zhuang, H., Gamble, G. R., and Bowker, B. C. (2020). Effect of spaghetti meat abnormality on broiler chicken breast meat composition and technological quality. Poult. Sci. 99, 1724-1733. doi: 10.1016/j.psj.2019. 10.069

Trocino, A., Piccirillo, A., Birolo, M., Radaelli, G., Bertotto, D., Filiou, E., et al. (2015). Effect of genotype, gender and feed restriction on growth, meat quality and the occurrence of white striping and wooden breast in broiler chickens. Poult. Sci. 94, 2996-3004. doi: 10.3382/ps/pev296

Xing, T., Zhao, X., Cai, L., Guanghong, Z., and Xu, X. (2017). Effect of salt content on gelation of normal and wooden breast myopathy chicken pectoralis major meat batters. Int. J. Food Sci. Technol. 52, 2068-2077. doi: 10.1111/ijfs.13485

Young, J. F., and Rasmussen, M. K. (2020). Differentially expressed marker genes and glycogen levels in pectoralis major of Ross 308 broilers with wooden breast syndrome indicates stress, inflammation and hypoxic conditions. Food Chem. Mol. Sci. 1:100001. doi: 10.1016/j.fochms.2020.100001

Yu, X., Feng, Y., Bowker, B., and Zhuang, H. (2020). Expressible fluid measurements of broiler breast meat affected by emerging muscle abnormalities. LWT 133:110110. doi: 10.1016/j.lwt.2020.110110

Zampiga, M., Laghi, L., Petracci, M., Zhu, C., Meluzzi, A., Dridi, S., et al. (2018). Effect of dietary arginine to lysine ratios on productive performance, meat quality, plasma and muscle metabolomics profile in fast-growing broiler chickens. J. Anim. Sci. Biotechnol. 9:79. doi: 10.1186/s40104-018-0294-5

Zampiga, M., Soglia, F., Baldi, G., Petracci, M., Strasburg, G. M., and Sirri, F. (2020). Muscle abnormalities and meat quality consequences in modern turkey hybrids. Front. Physiol. 11:554. doi: 10.3389/fphys.2020.00554

Conflict of Interest: The authors declare that the research was conducted in the absence of any commercial or financial relationships that could be construed as a potential conflict of interest.

The reviewer EP declared a past co-authorship, with one of the authors, FS, to the handling editor.

Copyright (C) 2021 Baldi, Soglia and Petracci. This is an open-access article distributed under the terms of the Creative Commons Attribution License (CC BY). The use, distribution or reproduction in other forums is permitted, provided the original author(s) and the copyright owner(s) are credited and that the original publication in this journal is cited, in accordance with accepted academic practice. No use, distribution or reproduction is permitted which does not comply with these terms. 